\title{
Encsergy
}

\section{Highly Mobile Large Polarons in Black Phase $\mathrm{CsPbl}_{3}$}

Heng Zhang," Elke Debroye," Julian A. Steele, Maarten B. J. Roeffaers, Johan Hofkens, Hai I. Wang,* and Mischa Bonn

Cite This: ACS Energy Lett. 2021, 6, 568-573

Read Online

ACCESS

Wlll Metrics \& More

Article Recommendations

Supporting Information

ABSTRACT: We report the band-like transport of photogenerated charge carriers within all-inorganic black $\gamma$-phase $\mathrm{CsPbI}_{3}(\gamma \text {-CsPbI })_{3}$ thin films, with local mobilities up to $270 \pm 44 \mathrm{~cm}^{2} \mathrm{~V}^{-1} \mathrm{~s}^{-1}$ recorded using terahertz ( $\mathrm{THz}$ ) spectroscopy at room temperature. Temperature-dependent, highfrequency photoconductivity measurements indicate that large polaron formation governs charge carrier transport, following the Feynman polaron model. The mobility values derived using $\mathrm{THz}$ spectroscopy are nearly 1 order of magnitude higher than that reported for hybrid organicinorganic lead halide perovskites and approach the theoretical limit for polarons scattering from longitudinal optical (LO) phonons. Our results identify $\gamma-\mathrm{CsPbI}_{3}$ as a fascinating all-inorganic perovskite semiconductor with high charge carrier mobility for optoelectronics and reveal the effect of polaron formation on charge transport properties.

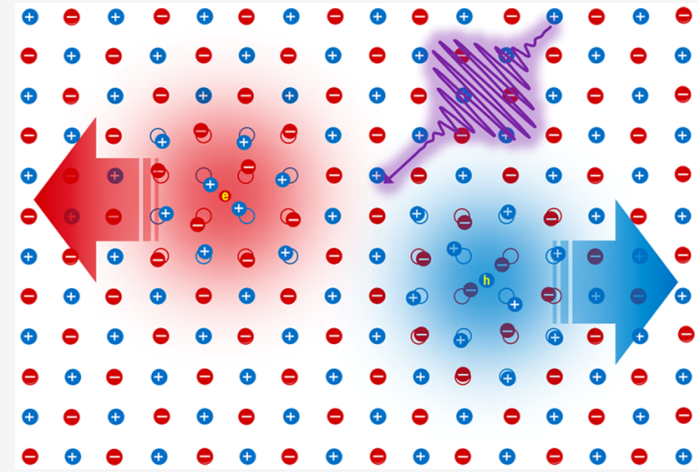

nation, carrier multiplication, etc.) on the picosecond (ps) time scale following photoexcitation. ${ }^{19-22}$ While the inferred carrier lifetimes in these reports are of great interest for developing efficient devices, the charge transport properties, such as the mobility of photogenerated carriers, a vital figure of merit for photovoltaic applications, are yet to be revealed.

Due to the "soft" and polar nature of lead halide perovskite crystals, photogenerated charge carriers have been shown to polarize the lattice and induce atomic displacement of the ions from their equilibrium positions. ${ }^{23,24}$ The charge carriers, together with the associated local lattice deformation, can be described as a polaron quasi-particle. ${ }^{25}$ Several recent experiments have provided spectroscopic evidence of polaron formation in perovskites, ${ }^{26-32}$ by, e.g., probing subpicosecond lattice or vibrational dynamics. Polaron formation has been reported to be responsible for several of the fascinating optoelectronic properties in LHPs, including long-lived hot carriers and slow carrier recombination processes. ${ }^{33,34}$ Exploring the influence of polaron formation on the photogenerated charge carrier transport will be essential for

Received: November 27, 2020

Accepted: January 13, 2021

Published: January 20, 2021

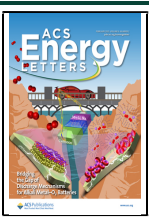
yet its photophysical details remain largely unexplored. Initial optical studies focused on understanding the evolution of charge carriers (charge separation and recombi- 
(a)

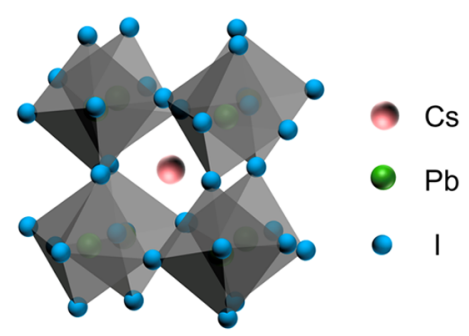

(c)

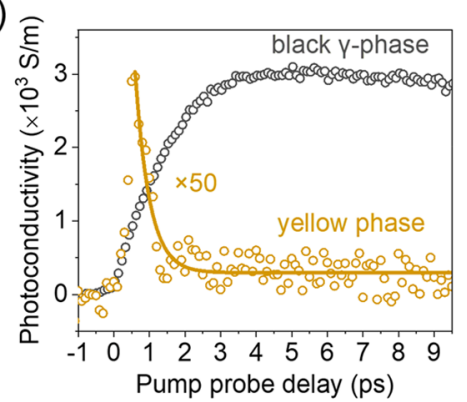

(b)

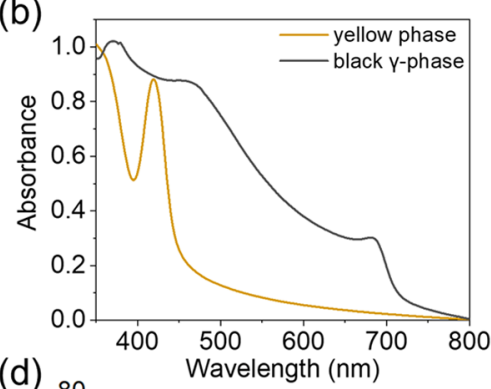

(d)

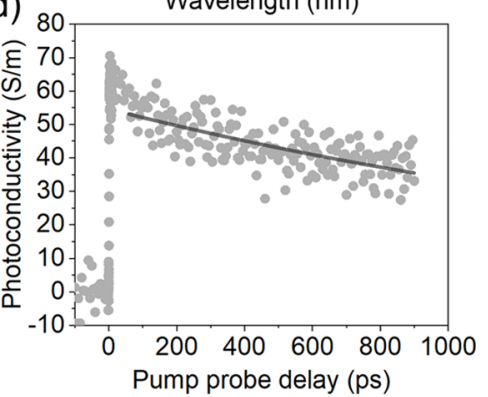

Figure 1. (a) Schematic representation of the black $\gamma$-CsPbI ${ }_{3}$ crystal structure. (b) Absorption spectra of yellow phase and $\gamma$-CsPbI ${ }_{3}$. (c) $\mathrm{THz}$ photoconductivity comparison for $\gamma-\mathrm{CsPbI}_{3}$ and yellow phase at the same absorbed photon density of $5.81 \times 10^{13} \mathrm{~cm}^{-2}$. The solid line represents a single exponential fit with an offset. (d) Photoconductivity dynamics of $\gamma-\mathrm{CsPbI}_{3}$ at the absorbed photon density of $1.67 \times 10^{12}$ $\mathrm{cm}^{-2}$. The solid line is an exponential fit that yields a decay time of $\sim 2 \mathrm{~ns}$.

fundamentally understanding $\mathrm{CsPbI}_{3}$-based optical device design and function.

Here, we report on the temperature-dependent transport properties of photogenerated charge carriers in black phase $\gamma$ $\mathrm{CsPb}_{3}$ thin films employing ultrafast terahertz spectroscopy. The remarkably high charge carrier mobility in $\gamma$-CsPbI 3 of $\sim 270 \pm 44 \mathrm{~cm}^{2} \mathrm{~V}^{-1} \mathrm{~s}^{-1}$ at room temperature approaches the theoretical limit, based on the Feynman polaron model of charge-LO phonon interaction. This result indicates that grain boundaries and defects do not play critical roles in determining the charge mobility in our samples. As such, we expect the mobility reported here for a polycrystalline sample to be that in single $\gamma$ - $\mathrm{CsPbI}_{3}$ crystals. Furthermore, this value is almost an order of magnitude higher than that for hybrid LHPs.

Recently, Steele et al. ${ }^{7}$ examined the thermal phase relations of polymorphic $\mathrm{CsPbI}_{3}$ thin films and demonstrated the formation of a metastable, strained $\gamma-\mathrm{CsPbI}_{3}$ perovskite thin film at RT. Hence, RT $\gamma$-CsPbI ${ }_{3}$ polycrystalline thin films were prepared following the reported method (see details in SI). ${ }^{7}$ The $\gamma$ - $\mathrm{CsPbI}_{3}$ persists at RT long enough to finalize the spectroscopic study when maintained under a nitrogen atmosphere or under vacuum conditions-supported by stable photoconductivity properties. A schematic illustration of the orthorhombic crystal structure of the $\gamma-\mathrm{CsPbI}_{3}$ is shown in Figure 1a. The RT UV-vis absorption spectra of the same sample before (yellow phase) and after (black $\gamma$-phase) the thermal-driven phase transition are shown in Figure $1 \mathrm{~b}$. Compared to the yellow phase, the $\gamma-\mathrm{CsPbI}_{3}$ exhibits much stronger absorption across the UV-vis spectrum with a direct bandgap around the expected $1.75 \mathrm{eV}$ (Figure S1).

To investigate the dynamics and transport properties of photogenerated carriers in polycrystalline $\gamma-\mathrm{CsPbI}_{3}$ thin films, we employed ultrafast optical pump- $\mathrm{THz}$ probe (OPTP) spectroscopy. ${ }^{35}$ In a typical OPTP measurement, a $400 \mathrm{~nm}$, $\sim 50 \mathrm{fs}(\mathrm{fs})$ laser pulse promotes electrons from the valence to the conduction band. After a specific delay time, a collinearly propagating $\mathrm{THz}$ pulse with $\sim 1$ ps duration probes the photoconductivity $\sigma$ of the sample. The photogenerated free charges can be accelerated by the oscillating $\mathrm{THz}$ electric field $(E)$, resulting in the attenuation of $E$. The relative change of the THz field, defined as $-\Delta E / E$, is proportional to $\sigma$ which is determined by the carrier density, $n$, and charge mobility, $\mu$, following: $\sigma=n e \mu$ ( $e$ the elementary charge).

In Figure 1c, the photoconductivity dynamics at the same absorbed photon density are compared for yellow phase and $\gamma$ $\mathrm{CsPb}_{3}$ thin films. The photoconductivity rise in $\gamma-\mathrm{CsPbI}_{3}$ in the first $2-3$ ps is attributed to hot carrier cooling. ${ }^{36}$ Relative to the yellow phase, the charge carriers in the black phase possess a much longer lifetime (ns vs subps, see Figure $1 \mathrm{c}$ and d) and a $\sim 50$ times higher peak conductivity. The yellow phase's fast photoconductivity decay is assigned to ultrafast charge trapping due to the high defect density. This high defect density may be partially responsible for the low photoconductivity of yellow phase $\mathrm{CsPbI}_{3}$. The comparative study demonstrates the superior photophysical and charge transport properties of $\gamma-\mathrm{CsPbI}_{3}$ and its suitability for photovoltaics. The data in Figure 1d were recorded at sufficiently low excitation fluence to avoid higher-order recombination effects. Detailed fluence-dependent results (Figure S2) and analysis can be found in the SI, including the corresponding charge recombination fitting by the rate equation and a comparison of the rate constants with previous reports. On the basis of the analysis (see SI), we find that the monomolecular trapping rate is enhanced ( $\sim 40$ times higher) compared to $\mathrm{MAPbI}_{3}$ samples reported by Herz. ${ }^{37}$ This indicates a relatively high defect density present in our samples.

To determine the charge carrier mobility, an important figure of merit within photovoltaic devices, we conduct a timedomain spectroscopic (TDS) analysis for the $\gamma-\mathrm{CsPb}_{3}$ thin film at a given pump-probe delay time. TDS provides frequency-resolved complex conductivity spectra as exemplarily shown in Figure 2a: 4 ps, 124 ps, and 524 ps after photoexcitation (for TDS measurement and data analysis details, see SI). Both the real and imaginary parts are positive 
(a)

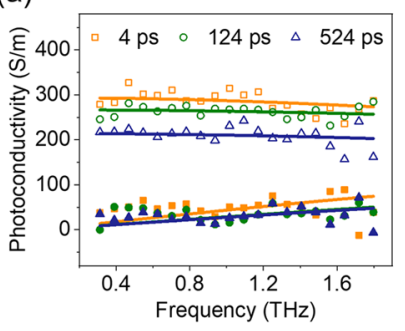

(b)

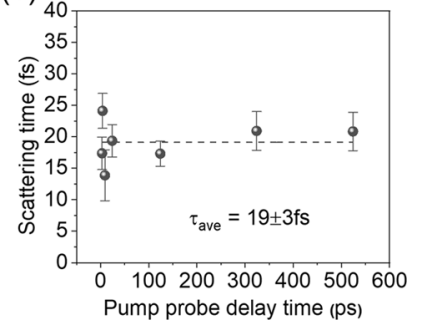

Figure 2. (a) Real (open symbols) and imaginary (solid symbols) components of the $\mathrm{THz}$ frequency-domain conductivity measured 4 ps, 124 ps, and 524 ps after photoexcitation with a photon density of $5.9 \times 10^{12} \mathrm{~cm}^{-2}$ at RT in a vacuum. The solid lines represent Drude model fits. (b) Scattering times measured at different pump-probe time delays. The dashed line is the average of all scattering times.

and show negative and positive dispersion within the $0.25-1.8$ $\mathrm{THz}$ bandwidth of the THz pulse, respectively. The Drude model (lines in Figure 2a) provides a quantitative description of the data: ${ }^{35,38}$

$$
\sigma(\omega)=\frac{\omega_{p}^{2} \varepsilon_{0} \tau_{s}}{1-i \omega \tau_{s}}
$$

Here, $\omega_{p}$ is the plasma frequency, $\varepsilon_{0}$ the vacuum permittivity, $\tau_{s}$ the scattering time and $\omega$ the angular frequency. From the good agreement, we infer the mean charge scattering time $\tau_{s}$ to be $19 \pm 3$ fs based on measurements spanning time delays from 3 to $\sim 550$ ps, as shown in Figure 2b. Remarkably, despite the relatively high defect density (manifested by the large firstorder recombination constant $k_{1}$, see SI), the obtained charge scattering time in black phase $\mathrm{CsPbI}_{3}$ is found to be $\sim 5$ times longer than that in hybrid LHP samples with the same polycrystalline nature in the film as our $\gamma-\mathrm{CsPbI}_{3}$. For example, a scattering time of $4 \pm 0.5$ fs in $\mathrm{MAPbI}_{3}$ films has been reported previously. ${ }^{39}$

To gain additional insight into the charge carrier transport, we conducted temperature $(T)$ dependent $\mathrm{THz}$ conductivity measurements between $77 \mathrm{~K}$ and RT. Previous THz-based measurements on hybrid perovskites found a $T^{-1.5}$-dependence of the mobility, which was interpreted by free electron-acoustic phonon scattering. ${ }^{39-42}$ Meanwhile, the formation of large polarons (i.e., with lattice distortion beyond several lattice constants) has shown to occur on subpicosecond time scales in all-inorganic perovskites, including $\mathrm{CsPbI}_{3} \cdot{ }^{36,43}$ Large polarons undergo band-like transport, with the mobility decreasing with increasing temperature, i.e., $d \mu / d T<0$. Frost ${ }^{24}$ has calculated the charge mobility in perovskites, including all-inorganic $\mathrm{CsPb}_{3}$, based on the Feynman polaron model, assuming the dominant role of LO phonon scattering for polaron transport. In addition, the polaron mobility is found to increase nonlinearly with lowering $T$. For the $T$ range studied here, Frost predicts that $\mu \propto T^{-\beta}$, with $\beta \sim 0.5 .^{24}$ As shown in Figure 3 , we present both the photoconductivity of charge carriers based on the OPTP study, and the charge scattering time extracted from the Drude model, as a function of temperature. The general trend for both parameters is consistent with the transport of large polarons. For the experimental data in Figure 3, we find $\beta \sim 0.40$ for the scattering time and $\beta \sim 0.77$ for the photoconductivity. This reasonable agreement between our experimental results and theoretical predictions supports the notion of large polaron transport, limited by polaron-LO

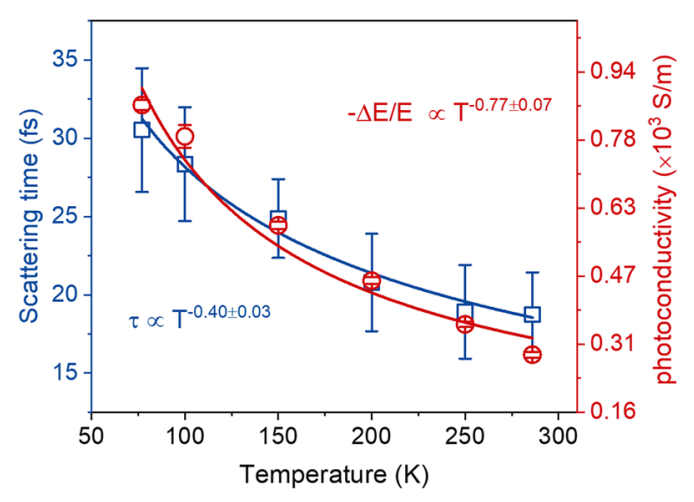

Figure 3. Temperature-dependent scattering time and photoconductivity (proportional to $-\Delta E / E$ ). In this study, the incident photon density and wavelength for the optical excitation pulse is fixed $\left(5.9 \times 10^{12} \mathrm{~cm}^{-2}\right.$ for $400 \mathrm{~nm}$ excitation $)$. The solid lines are power-law fits.

phonon scattering in $\gamma$-CsPbI 3 . The large polaron formation can also explain the observed high defect tolerance in our sample. $^{33,34}$

To quantify the charge carrier mobility, $\mu$, from the scattering time using the relation $\mu=\frac{e \tau_{s}}{m^{*}}$, we need knowledge of the effective mass of the photogenerated charge carriers $m^{*}$. This can be obtained from band structure calculations, but other renormalization factors, e.g., polaron formation, need to be considered. Large polarons can exhibit band-like transport with an increased effective mass, $m^{*}$, relative to the bare band mass, $m_{\mathrm{b}}^{*}$, of the solids, ${ }^{44}$ following: ${ }^{45}$

$$
m^{*}=m_{\mathrm{b}}^{*}\left(1+\frac{\alpha}{6}+\frac{\alpha^{2}}{40}+\ldots\right)
$$

Here, $\alpha$ is the dimensionless Fröhlich electron-phonon coupling constant, defined as ${ }^{46}$

$$
\alpha=\frac{e^{2}}{4 \pi \hbar}\left(\frac{1}{\varepsilon_{\mathrm{opt}}}-\frac{1}{\varepsilon_{\mathrm{s}}}\right) \sqrt{\frac{m_{\mathrm{b}}^{*}}{2 \hbar \omega_{\mathrm{LO}}}}
$$

where $\varepsilon_{o p t}$ and $\varepsilon_{s}$ are respectively the optical and static dielectric constants (in units of vacuum permittivity $\varepsilon_{0}$ ), $\omega_{L O}$ is the effective LO phonon angular frequency of the coupled modes, and $\hbar$ is the reduced Planck constant. Notably, previous theoretical calculations have reported very similar properties for $\alpha$ - and $\gamma$-black phases in terms of electronic structure and electron-phonon coupling strength. ${ }^{47,48}$ Therefore, here, we estimate the Fröhlich electron-phonon coupling constant of the $\gamma$-phase, using the widely reported dielectric properties and vibrational modes of the $\alpha$-phase $\left(\varepsilon_{o p t}=6.1 \varepsilon_{0}\right.$, $\varepsilon_{\mathrm{s}}=18.1 \varepsilon_{0}, \omega_{L O}=16.15 \mathrm{THz}$, respectively), based on theoretical studies. ${ }^{24}$ These values are in line with previously reported experimental results for black phase $\mathrm{CsPbI}_{3}{ }^{49,50}$ We calculate $\alpha$ to be 1.23 . The small value of $\alpha$ is consistent with the formation of large polarons $(\alpha<6)$ in the $\gamma-\mathrm{CsPbI}_{3}$ as well. $^{51}$ Using the band effective mass, $m_{\mathrm{b}}^{*}$, from DFT calculations $\left(\sim 0.1 m_{0}\right.$, in which $m_{0}$ is the rest mass of an electron $\left.{ }^{48,52}\right), m^{*}$ is calculated to be $0.124 m_{0}{ }^{53}$ With this value, the charge carrier mobility amounts to $\sim 270 \pm 44 \mathrm{~cm}^{2}$ $\mathrm{V}^{-1} \mathrm{~s}^{-1}$, which is nearly an order of magnitude higher than other hybrid LHP polycrystalline thin films, as summarized in Table 1 . The high mobility of the large polaron originates primarily from the long charge scattering time. Previous 
Table 1. Comparison of the Mobility Measured in $\gamma-\mathrm{CsPbI}_{3}$ Wtih That from Calculations and Measurements in Other Reports for Hybrid LHPs

$\begin{array}{ccc}\text { material (polycrystalline thin film) } & \text { mobility }\left(\mathrm{cm}^{2} \mathrm{~V}^{-1} \mathrm{~s}^{-1}\right) & \text { technique } \\ \mathrm{CsPbI}_{3} \text { (this work) } & 270 \pm 44 & \mathrm{THz} \\ \mathrm{CsPbI}_{3} & 344 & \text { calculation }^{24} \\ \mathrm{MAPbI}_{3} & \sim 27 & \mathrm{THz}^{39} \\ \mathrm{MAPbI}_{3} & \sim 20-75 & \mathrm{TRMC}^{54, a} \\ \mathrm{MAPbI}_{3} & \sim 25 & \mathrm{THz}^{55} \\ \mathrm{FAPbI}_{3} & \sim 75 & \mathrm{THz}^{56} \\ \mathrm{FAPbI}_{3} & \sim 27 & \mathrm{THz}^{57} \\ \mathrm{MAPbI}_{3} \text { (single crystal) } & \sim 620 & \mathrm{THz}^{38}\end{array}$

$a_{\mathrm{TRMC}}=$ time-resolved microwave conductivity.

theoretical studies by Zheng et al. revealed that dynamic disorder caused by the organic cations can cause localization of charge carriers and thus reduce the mobility of large polarons in LHPs. ${ }^{23}$ Our observation of a substantial enhancement in charge carrier mobility in all-inorganic perovskites compared to their organic-inorganic hybrid counterparts supports this conclusion. Furthermore, based on the lifetime inferred from the dynamics and the estimated mobility, the diffusion length of photogenerated polarons exceeds $1 \mu \mathrm{m}$ (see the SI for more details). This result illustrates the great potential of the $\gamma$ $\mathrm{CsPbI}_{3}$ for photovoltaic applications.

To further verify the high mobility of charge carriers in $\gamma$ $\mathrm{CsPb}_{3}$, we conducted a second estimate of the mobility from the amplitude of the OPTP data as exhibited in Figure 1c,d (see SI for more details). This is distinct from the first method, which relies on the dispersion (frequency dependence) of the complex photoconductivity. Briefly, $-\Delta E / E$ is proportional to the photoconductivity, $\sigma$, of the free charge carriers, and $\mu=\sigma$ / (en). The photogenerated carrier density, $n$, can be obtained from the Drude model, since $\omega_{\mathrm{p}}^{2}=\frac{e^{2} \cdot n}{\varepsilon_{0} \cdot m^{*}}$. This estimate reveals a mobility of $258 \pm 6 \mathrm{~cm}^{2} \mathrm{~V}^{-1} \mathrm{~s}^{-1}$. This value is in good agreement with the polaron-based mobility estimation made from the TDS dispersion analysis, supporting the estimates of the electron-phonon coupling constant and polaron effective mass giving rise to highly mobile large polarons in black phase $\mathrm{CsPbI}_{3}$.

Furthermore, based on the code developed by Frost and using the band effective mass $\left(0.1 m_{0}\right)$ given by previous DFT studies, ${ }^{48}$ we calculate the maximum polaron mobility to be $344 \mathrm{~cm}^{2} \mathrm{~V}^{-1} \mathrm{~s}^{-1}$ at RT for $\gamma-\mathrm{CsPbI}_{3}$. Thus, the experimentally determined mobility in our sample reaches $\sim 80 \%$ of the theoretical limit. The remaining difference between the experimental and theoretical results may be due to the contributions from defect scattering or dielectric drag effects in perovskites. ${ }^{51}$

In conclusion, we have performed a systematic $\mathrm{THz}$ spectroscopic study of $\gamma-\mathrm{CsPbI}_{3}$ and revealed a remarkably high charge carrier mobility in the material, up to $270 \pm 44$ $\mathrm{cm}^{2} \mathrm{~V}^{-1} \mathrm{~s}^{-1}$, which is nearly 1 order of magnitude higher than that of conventional hybrid lead halide perovskites. We further provide evidence for large polaron formation dictating the transport properties and $T$-dependent conductivity of the photogenerated carriers. Our results highlight the black $\gamma$ phase $\mathrm{CsPb}_{3}$ as a fascinating all-inorganic perovskite material class for efficient optoelectronics.

\section{ASSOCIATED CONTENT}

\section{Supporting Information}

The Supporting Information is available free of charge at https://pubs.acs.org/doi/10.1021/acsenergylett.0c02482.

$\mathrm{CsPbI}_{3}$ film preparation, estimation of the direct bandgap energy in black phase $\mathrm{CsPb}_{3}$, optical pump$\mathrm{THz}$ probe spectroscopy, recombination rate equation fitting to the photoconductivity transient dynamics, comparison of recombination rate constants, determination of charge carrier lifetime and diffusion length, mobility estimation from optical pump- $\mathrm{THz}$ probe measurement (PDF)

\section{AUTHOR INFORMATION}

\section{Corresponding Author}

Hai I. Wang - Max Planck Institute for Polymer Research, 55128 Mainz, Germany; 이이.org/0000-0003-0940-

3984; Email: wanghai@mpip-mainz.mpg.de

\section{Authors}

Heng Zhang - Max Planck Institute for Polymer Research, 55128 Mainz, Germany; 이이.org/0000-0002-51757367

Elke Debroye - Department of Chemistry, KU Leuven, 3001 Leuven, Belgium; 이잉.org/0000-0003-1087-4759

Julian A. Steele - cMACS, Department of Microbial and Molecular Systems, KU Leuven, 3001 Leuven, Belgium; (1) orcid.org/0000-0001-7982-4413

Maarten B. J. Roeffaers - cMACS, Department of Microbial and Molecular Systems, KU Leuven, 3001 Leuven, Belgium; (1) orcid.org/0000-0001-6582-6514

Johan Hofkens - Department of Chemistry, KU Leuven, 3001 Leuven, Belgium; Max Planck Institute for Polymer Research, 55128 Mainz, Germany; 이이.org/0000-0002-91010567

Mischa Bonn - Max Planck Institute for Polymer Research, 55128 Mainz, Germany; 이이이.org/0000-0001-68518453

Complete contact information is available at:

https://pubs.acs.org/10.1021/acsenergylett.0c02482

\section{Author Contributions}

"Contributed equally to this work.

\section{Notes}

The authors declare no competing financial interest.

\section{ACKNOWLEDGMENTS}

We thank Keno Krewer, Wenhao Zheng, Shuai Fu, and Lucia Di Virgilio for their help and discussions on the data analysis. The authors acknowledge financial support from the Research Foundation - Flanders (FWO) through postdoctoral fellowships to E.D. and J.A.S. (FWO grant nos. 12O3719N and $12 \mathrm{Y} 7221 \mathrm{~N}$ ) and research projects to J.H. and M.B.J.R. (FWO grant nos. S002019N, G098319N, 1514220N, and ZW15 09GOH6316), the Flemish government through long-term structural funding Methusalem (CASAS2, Meth/15/04), the Hercules Foundation (HER/11/14), the Belgian Federal Science Policy Office (IAP-PH05), the KU Leuven Research Fund (C14/19/079), and the MPI financial support to J.H. as an MPI fellow. 


\section{REFERENCES}

(1) Green, M. A.; Dunlop, E. D.; Hohl-Ebinger, J.; Yoshita, M.; Kopidakis, N.; Ho-Baillie, A. W. Y. Solar cell efficiency tables (Version 55). Prog. Photovoltaics 2020, 28 (1), 3-15.

(2) Green, M. A.; Ho-Baillie, A.; Snaith, H. J. The emergence of perovskite solar cells. Nat. Photonics 2014, 8 (7), 506-514.

(3) Stranks, S. D.; Eperon, G. E.; Grancini, G.; Menelaou, C.; Alcocer, M. J.; Leijtens, T.; Herz, L. M.; Petrozza, A.; Snaith, H. J. Electron-hole diffusion lengths exceeding 1 micrometer in an organometal trihalide perovskite absorber. Science 2013, 342 (6156), 341-344.

(4) Dong, Q.; Fang, Y.; Shao, Y.; Mulligan, P.; Qiu, J.; Cao, L.; Huang, J. Electron-hole diffusion lengths $>175 \mu \mathrm{m}$ in solution-grown CH3NH3PbI3 single crystals. Science 2015, 347 (6225), 967-970.

(5) Kang, J.; Wang, L. W. High Defect Tolerance in Lead Halide Perovskite CsPbBr3. J. Phys. Chem. Lett. 2017, 8 (2), 489-493.

(6) Huang, J.; Yuan, Y.; Shao, Y.; Yan, Y. Understanding the physical properties of hybrid perovskites for photovoltaic applications. Nature Reviews Materials 2017, 2 (7), 1-19.

(7) Steele, J. A.; Jin, H.; Dovgaliuk, I.; Berger, R. F.; Braeckevelt, T.; Yuan, H.; Martin, C.; Solano, E.; Lejaeghere, K.; Rogge, S. M. J.; Notebaert, C.; Vandezande, W.; Janssen, K. P. F.; Goderis, B.; Debroye, E.; Wang, Y. K.; Dong, Y.; Ma, D.; Saidaminov, M.; Tan, H.; Lu, Z.; Dyadkin, V.; Chernyshov, D.; Van Speybroeck, V.; Sargent, E. H.; Hofkens, J.; Roeffaers, M. B. J. Thermal unequilibrium of strained black CsPbI3 thin films. Science 2019, 365 (6454), 679-684.

(8) Conings, B.; Drijkoningen, J.; Gauquelin, N.; Babayigit, A.; D’Haen, J.; D’Olieslaeger, L.; Ethirajan, A.; Verbeeck, J.; Manca, J.; Mosconi, E.; Angelis, F. D.; Boyen, H.-G. Intrinsic Thermal Instability of Methylammonium Lead Trihalide Perovskite. Adv. Energy Mater. 2015, 5 (15), 1500477

(9) Christians, J. A.; Miranda Herrera, P. A.; Kamat, P. V. Transformation of the excited state and photovoltaic efficiency of $\mathrm{CH} 3 \mathrm{NH} 3 \mathrm{PbI} 3$ perovskite upon controlled exposure to humidified air. J. Am. Chem. Soc. 2015, 137 (4), 1530-1538.

(10) Eperon, G. E.; Stranks, S. D.; Menelaou, C.; Johnston, M. B.; Herz, L. M.; Snaith, H. J. Formamidinium lead trihalide: a broadly tunable perovskite for efficient planar heterojunction solar cells. Energy Environ. Sci. 2014, 7 (3), 982-988.

(11) Kulbak, M.; Gupta, S.; Kedem, N.; Levine, I.; Bendikov, T.; Hodes, G.; Cahen, D. Cesium Enhances Long-Term Stability of Lead Bromide Perovskite-Based Solar Cells. J. Phys. Chem. Lett. 2016, 7 (1), $167-172$.

(12) Eperon, G. E.; Paternò, G. M.; Sutton, R. J.; Zampetti, A.; Haghighirad, A. A.; Cacialli, F.; Snaith, H. J. Inorganic caesium lead iodide perovskite solar cells. J. Mater. Chem. A 2015, 3 (39), 1968819695.

(13) Wang, Y.; Dar, M. I.; Ono, L. K.; Zhang, T.; Kan, M.; Li, Y.; Zhang, L.; Wang, X.; Yang, Y.; Gao, X.; Qi, Y.; Gratzel, M.; Zhao, Y. Thermodynamically stabilized $\beta$-CsPbI3-based perovskite solar cells with efficiencies> 18\%. Science 2019, 365 (6453), 591-595.

(14) Swarnkar, A.; Marshall, A. R.; Sanehira, E. M.; Chernomordik, B. D.; Moore, D. T.; Christians, J. A.; Chakrabarti, T.; Luther, J. M. Quantum dot-induced phase stabilization of $\alpha$-CsPbI3 perovskite for high-efficiency photovoltaics. Science 2016, 354 (6308), 92-95.

(15) Li, B.; Zhang, Y.; Fu, L.; Yu, T.; Zhou, S.; Zhang, L.; Yin, L. Surface passivation engineering strategy to fully-inorganic cubic CsPbI3 perovskites for high-performance solar cells. Nat. Commun. 2018, 9 (1), 1-8.

(16) Wang, K.; Jin, Z.; Liang, L.; Bian, H.; Bai, D.; Wang, H.; Zhang, J.; Wang, Q.; Liu, S. All-inorganic cesium lead iodide perovskite solar cells with stabilized efficiency beyond 15. Nat. Commun. 2018, 9 (1), $1-8$.

(17) Wang, P.; Zhang, X.; Zhou, Y.; Jiang, Q.; Ye, Q.; Chu, Z.; Li, X.; Yang, X.; Yin, Z.; You, J. Solvent-controlled growth of inorganic perovskite films in dry environment for efficient and stable solar cells. Nat. Commun. 2018, 9 (1), 1-7.

(18) Wang, Y.; Zhang, T.; Kan, M.; Zhao, Y. Bifunctional Stabilization of All-Inorganic alpha-CsPbI3 Perovskite for $17 \%$
Efficiency Photovoltaics. J. Am. Chem. Soc. 2018, 140 (39), 1234512348.

(19) Shang, Q.; Piercy, B. D.; Losego, M. D.; Lian, T. Effect of Surface Ligand on Charge Separation and Recombination at CsPbI3 Perovskite Quantum Dot/TiO2 Interfaces. J. Phys. Chem. C 2019, 123 (35), 21415-21421.

(20) Liu, F.; Zhang, Y.; Ding, C.; Toyoda, T.; Ogomi, Y.; Ripolles, T. S.; Hayase, S.; Minemoto, T.; Yoshino, K.; Dai, S.; Shen, Q. Ultrafast Electron Injection from Photoexcited Perovskite CsPbI3 QDs into $\mathrm{TiO} 2$ Nanoparticles with Injection Efficiency near 99. J. Phys. Chem. Lett. 2018, 9 (2), 294-297.

(21) Cong, M.; Yang, B.; Chen, J.; Hong, F.; Yang, S.; Deng, W.; Han, K. Carrier Multiplication and Hot-Carrier Cooling Dynamics in Quantum-Confined CsPbI3 Perovskite Nanocrystals. J. Phys. Chem. Lett. 2020, 11 (5), 1921-1926.

(22) de Weerd, C.; Gomez, L.; Capretti, A.; Lebrun, D. M.; Matsubara, E.; Lin, J.; Ashida, M.; Spoor, F. C. M.; Siebbeles, L. D. A.; Houtepen, A. J.; Suenaga, K.; Fujiwara, Y.; Gregorkiewicz, T. Efficient carrier multiplication in $\mathrm{CsPbI} 3$ perovskite nanocrystals. Nat. Commun. 2018, 9 (1), 1-9.

(23) Zheng, F.; Wang, L.-w. Large polaron formation and its effect on electron transport in hybrid perovskites. Energy Environ. Sci. 2019, 12 (4), 1219-1230.

(24) Frost, J. M. Calculating polaron mobility in halide perovskites. Phys. Rev. B: Condens. Matter Mater. Phys. 2017, 96 (19), 195202.

(25) Emin, D. Optical properties of large and small polarons and bipolarons. Phys. Rev. B: Condens. Matter Mater. Phys. 1993, 48 (18), 13691-13702.

(26) Ghosh, T.; Aharon, S.; Etgar, L.; Ruhman, S. Free Carrier Emergence and Onset of Electron-Phonon Coupling in Methylammonium Lead Halide Perovskite Films. J. Am. Chem. Soc. 2017, 139 (50), 18262-18270.

(27) Batignani, G.; Fumero, G.; Srimath Kandada, A. R.; Cerullo, G.; Gandini, M.; Ferrante, C.; Petrozza, A.; Scopigno, T. Probing femtosecond lattice displacement upon photo-carrier generation in lead halide perovskite. Nat. Commun. 2018, 9 (1), 1-5.

(28) Miyata, K.; Meggiolaro, D.; Trinh, M. T.; Joshi, P. P.; Mosconi, E.; Jones, S. C.; De Angelis, F.; Zhu, X.-Y. Large polarons in lead halide perovskites. Science advances 2017, 3 (8), No. e1701217.

(29) Steele, J. A.; Puech, P.; Keshavarz, M.; Yang, R.; Banerjee, S.; Debroye, E.; Kim, C. W.; Yuan, H.; Heo, N. H.; Vanacken, J.; Walsh, A.; Hofkens, J.; Roeffaers, M. B. J. Giant Electron-Phonon Coupling and Deep Conduction Band Resonance in Metal Halide Double Perovskite. ACS Nano 2018, 12 (8), 8081-8090.

(30) Keshavarz, M.; Debroye, E.; Ottesen, M.; Martin, C.; Zhang, H.; Fron, E.; Küchler, R.; Steele, J. A.; Bremholm, M.; Van de Vondel, J.; Wang, H. I.; Bonn, M.; Roeffaers, M. B. J.; Wiedmann, S.; Hofkens, J. Tuning the Structural and Optoelectronic Properties of Cs2AgBiBr6 Double-Perovskite Single Crystals through Alkali-Metal Substitution. Adv. Mater. 2020, 32 (40), 2001878.

(31) Steele, J. A.; Puech, P.; Monserrat, B.; Wu, B.; Yang, R. X.; Kirchartz, T.; Yuan, H.; Fleury, G.; Giovanni, D.; Fron, E.; Keshavarz, M.; Debroye, E.; Zhou, G.; Sum, T. C.; Walsh, A.; Hofkens, J.; Roeffaers, M. B. J. Role of Electron-Phonon Coupling in the Thermal Evolution of Bulk Rashba-Like Spin-Split Lead Halide Perovskites Exhibiting Dual-Band Photoluminescence. ACS Energy Letters 2019, 4 (9), 2205-2212.

(32) Guzelturk, B.; Winkler, T.; Van de Goor, T. W. J.; Smith, M. D.; Bourelle, S. A.; Feldmann, S.; Trigo, M.; Teitelbaum, S. W.; Steinruck, H. G.; de la Pena, G. A.; Alonso-Mori, R.; Zhu, D.; Sato, T.; Karunadasa, H. I.; Toney, M. F.; Deschler, F.; Lindenberg, A. M. Visualization of dynamic polaronic strain fields in hybrid lead halide perovskites. Nat. Mater. 2021, 1-6.

(33) Zhu, H.; Miyata, K.; Fu, Y.; Wang, J.; Joshi, P. P.; Niesner, D.; Williams, K. W.; Jin, S.; Zhu, X.-Y. Screening in crystalline liquids protects energetic carriers in hybrid perovskites. Science 2016, 353 (6306), 1409-1413. 
(34) Zhu, X. Y.; Podzorov, V. Charge Carriers in Hybrid OrganicInorganic Lead Halide Perovskites Might Be Protected as Large Polarons. J. Phys. Chem. Lett. 2015, 6 (23), 4758-4761.

(35) Ulbricht, R.; Hendry, E.; Shan, J.; Heinz, T. F.; Bonn, M. Carrier dynamics in semiconductors studied with time-resolved terahertz spectroscopy. Rev. Mod. Phys. 2011, 83 (2), 543-586.

(36) Bretschneider, S. A.; Ivanov, I.; Wang, H. I.; Miyata, K.; Zhu, X.; Bonn, M. Quantifying Polaron Formation and Charge Carrier Cooling in Lead-Iodide Perovskites. Adv. Mater. 2018, 30, No. e1707312.

(37) Wehrenfennig, C.; Eperon, G. E.; Johnston, M. B.; Snaith, H. J.; Herz, L. M. High Charge Carrier Mobilities and Lifetimes in Organolead Trihalide Perovskites. Adv. Mater. 2014, 26 (10), 15841589.

(38) Valverde-Chávez, D. A.; Ponseca, C. S.; Stoumpos, C. C.; Yartsev, A.; Kanatzidis, M. G.; Sundström, V.; Cooke, D. G. Intrinsic femtosecond charge generation dynamics in single crystal $\mathrm{CH} 3 \mathrm{NH} 3$ $\mathrm{PbI}$ 3. Energy Environ. Sci. 2015, 8 (12), 3700-3707.

(39) Karakus, M.; Jensen, S. A.; D’Angelo, F.; Turchinovich, D.; Bonn, M.; Canovas, E. Phonon-Electron Scattering Limits Free Charge Mobility in Methylammonium Lead Iodide Perovskites. J. Phys. Chem. Lett. 2015, 6 (24), 4991-4996.

(40) Milot, R. L.; Eperon, G. E.; Snaith, H. J.; Johnston, M. B.; Herz, L. M. Temperature-Dependent Charge-Carrier Dynamics in CH3NH3PbI3Perovskite Thin Films. Adv. Funct. Mater. 2015, 25 (39), 6218-6227.

(41) Savenije, T. J.; Ponseca, C. S., Jr.; Kunneman, L.; Abdellah, M.; Zheng, K.; Tian, Y.; Zhu, Q.; Canton, S. E.; Scheblykin, I. G.; Pullerits, T.; Yartsev, A.; Sundstrom, V. Thermally Activated Exciton Dissociation and Recombination Control the Carrier Dynamics in Organometal Halide Perovskite. J. Phys. Chem. Lett. 2014, 5 (13), 2189-2194.

(42) Keshavarz, M.; Wiedmann, S.; Yuan, H.; Debroye, E.; Roeffaers, M.; Hofkens, J. Light- and Temperature-Modulated Magneto-Transport in Organic-Inorganic Lead Halide Perovskites. ACS Energy Letters 2018, 3 (1), 39-45.

(43) Cinquanta, E.; Meggiolaro, D.; Motti, S. G.; Gandini, M.; Alcocer, M. J. P.; Akkerman, Q. A.; Vozzi, C.; Manna, L.; De Angelis, F.; Petrozza, A.; Stagira, S. Ultrafast THz Probe of Photoinduced Polarons in Lead-Halide Perovskites. Phys. Rev. Lett. 2019, 122 (16), 166601.

(44) Hendry, E.; Wang, F.; Shan, J.; Heinz, T. F.; Bonn, M. Electron transport in TiO2probed by $\mathrm{THz}$ time-domain spectroscopy. Phys. Rev. B: Condens. Matter Mater. Phys. 2004, 69 (8), 081101.

(45) Feynman, R. P. Slow Electrons in a Polar Crystal. Phys. Rev. 1955, 97 (3), 660-665.

(46) Fröhlich, H. Electrons in lattice fields. Adv. Phys. 1954, 3 (11), 325-361.

(47) Even, J.; Pedesseau, L.; Jancu, J.-M.; Katan, C. Importance of Spin-Orbit Coupling in Hybrid Organic/Inorganic Perovskites for Photovoltaic Applications. J. Phys. Chem. Lett. 2013, 4 (17), 29993005.

(48) Kang, Y.; Han, S. Intrinsic Carrier Mobility of Cesium Lead Halide Perovskites. Phys. Rev. Appl. 2018, 10 (4), 044013.

(49) Singh, R. K.; Kumar, R.; Jain, N.; Dash, S. R.; Singh, J.; Srivastava, A. Investigation of optical and dielectric properties of CsPbI3 inorganic lead iodide perovskite thin film. J. Taiwan Inst. Chem. Eng. 2019, 96, 538-542.

(50) Andrianov, A. V.; Aleshin, A. N.; Matyushkin, L. B. Terahertz Vibrational Modes in $\mathrm{CH} 3 \mathrm{NH} 3 \mathrm{PbI} 3$ and $\mathrm{CsPbI} 3$ Perovskite Films. JETP Lett. 2019, 109 (1), 28-32.

(51) Bonn, M.; Miyata, K.; Hendry, E.; Zhu, X. Y. Role of Dielectric Drag in Polaron Mobility in Lead Halide Perovskites. ACS Energy Letters 2017, 2 (11), 2555-2562.

(52) Sutton, R. J.; Filip, M. R.; Haghighirad, A. A.; Sakai, N.; Wenger, B.; Giustino, F.; Snaith, H. J. Cubic or Orthorhombic? Revealing the Crystal Structure of Metastable Black-Phase CsPbI3 by Theory and Experiment. ACS Energy Letters 2018, 3 (8), 1787-1794.
(53) Yang, Z.; Surrente, A.; Galkowski, K.; Miyata, A.; Portugall, O.; Sutton, R. J.; Haghighirad, A. A.; Snaith, H. J.; Maude, D. K.; Plochocka, P.; Nicholas, R. J. Impact of the Halide Cage on the Electronic Properties of Fully Inorganic Cesium Lead Halide Perovskites. ACS Energy Letters 2017, 2 (7), 1621-1627.

(54) Oga, H.; Saeki, A.; Ogomi, Y.; Hayase, S.; Seki, S. Improved understanding of the electronic and energetic landscapes of perovskite solar cells: high local charge carrier mobility, reduced recombination, and extremely shallow traps. J. Am. Chem. Soc. 2014, 136 (39), 13818-13825.

(55) Ponseca, C. S., Jr.; Savenije, T. J.; Abdellah, M.; Zheng, K.; Yartsev, A.; Pascher, T.; Harlang, T.; Chabera, P.; Pullerits, T.; Stepanov, A.; Wolf, J. P.; Sundstrom, V. Organometal halide perovskite solar cell materials rationalized: ultrafast charge generation, high and microsecond-long balanced mobilities, and slow recombination. J. Am. Chem. Soc. 2014, 136 (14), 5189-5192.

(56) Piatkowski, P.; Cohen, B.; Ponseca, C. S., Jr.; Salado, M.; Kazim, S.; Ahmad, S.; Sundstrom, V.; Douhal, A. Unraveling Charge Carriers Generation, Diffusion, and Recombination in Formamidinium Lead Triiodide Perovskite Polycrystalline Thin Film. J. Phys. Chem. Lett. 2016, 7 (1), 204-210.

(57) Rehman, W.; Milot, R. L.; Eperon, G. E.; Wehrenfennig, C.; Boland, J. L.; Snaith, H. J.; Johnston, M. B.; Herz, L. M. ChargeCarrier Dynamics and Mobilities in Formamidinium Lead MixedHalide Perovskites. Adv. Mater. 2015, 27 (48), 7938-7944. 